\title{
Phylogenetic Analysis of Astigmatid Mites Sarcoptes scabiei and Dermatophagoides farinae using ITS-2 as a Genetic Marker
}

Shumaila Naz ${ }^{1}$, Farhana Riaz Chaudhry ${ }^{2}$, Dilawar Abbas Rizvi', Muhammad Ismail ${ }^{4}$

\begin{abstract}
Objective: The coding of astigmatid mites based on their morphological and developmental characteristics often leads to uncertainty in the results. The ribosomal internal transcribed spacer (ITS-2) region, being highly conserved in eukaryotes is commonly employed as a barcode for identification of mite species. The present study was an attempt to characterize the gene sequences of astigmatid mites i.e. Sarcoptes scabiei (S. scabiei), Dermatophagoides farinae (D. farinae) using ITS-2 as a genetic marker.

Place and Duration of Study: The study was conducted at Department of Dermatology, Military Hospital (MH), Rawalpindi from September 2012 to October 2013.

Materials and Methods: In order to characterize relationship of astigmatid mites, the ITS-2 marker was successfully amplified and sequenced. The resulting ITS-2 gene sequences were aligned using Clustal W. MEGA 7 was used to construct phylogenetic tree of the aligned sequence.

Results: The phylogenetic tree showed an overall genetic distance of 0.53 indicating close genetic relationship among astigmatid mite species. Pairwise distance was calculated for the ITS-2 gene and low genetic diversity values were observed within S. scabiei and D. farinae that range from $0.003-0.008$ and $0.006-0.038$ respectively.

Conclusion: The study supports the view that the ITS-2 region can be used to identify morphologically difficult astigmatid mites but is not useful in characterization of different species based on the geographical distribution. This study has important implication in our understanding of the epidemiology of $S$. scabiei and $D$. farinae and development of control strategies in human transmission.
\end{abstract}

Key Words: Dermatophagoides Farina (Derf), House Dust Mite (HDM), ITS-2 region, Pakistan, Sarcoptes scabiei var. hominis.

How to cite this: Naz S, Chaudhry FR, Rizvi DA, Ismail M. Phylogenetic Analysis of Astigmatid Mites Sarcoptes scabiei and Dermatophagoides farinae using ITS-2 as a Genetic Marker. Life and Science. 2020; 1(2):55-59. doi: http://doi.org/10.37185/LnS.1.1.75

\section{Introduction}

Due to the ubiquitous nature of parasitic mites, they are known to infect invertebrates, vertebrates and plants. The parasitic infections are responsible for massive disease burden to humans and animals which in turn leads to higher levels of morbidity and mortality than any other infection. ${ }^{1,2,3}$ The

1,2 Department of Zoology

Pir Mehr Ali Shah Arid Agriculture University, Rawalpindi

Current Address: National University of Medical Sciences

Rawalpindi

${ }^{3}$ Department of Dermatology

Military Hospital, Rawalpindi

${ }^{4}$ Instituteof Biomedical and Genetic Engineering, Islamabad

Correspondence:

Dr. Shumaila Naz

Assistant Professor, Biological Sciences

National University of Medical Sciences, Rawalpindi

E-mail: shumaila.naz@numspak.edu.pk

Funding Source: HEC; Conflict of Interest: NIL

Received: Sept 26, 2019; Revised: Jan 21, 2020

Accepted: Feb 26, 2020 morphological identification of different developmental stages of mites often leads to uncertainty in the results. In the adult stage, mites are difficult to identify with high level of accuracy; complexity and similarity in taxonomy and morphology has made it challenging to differentiate between them with great precision. ${ }^{4,5,6}$

The limited availability of genomic data of astigmatid mites has implications related to genetic structure, epidemiology and monitoring protocols of the mites. ${ }^{7,8}$ Advances in molecular studies have allowed the exploration of the whole genomic sequence of mites ${ }^{9}$ which has enabled the recognition of taxonomically complex species and to comprehend associations between mite taxa. ${ }^{10,11}$

Phylogenetic relationships among different mite families are based on their morphological characteristics, allergen characterization, geographical distribution and physiological 
responses of mites to the environment and association with their hosts. ${ }^{12,13}$ Currently, molecular tools used to evaluate genetic diversification of species and to study phylogenetic interaction and relatedness among individual species are employing mitochondrial genes, ribosomal genes and internal transcribed spacers. ${ }^{14,15}$

In acarological research, the internal transcribed spacer ITS-2 gene has been used to study organization and kinship of a population, and to identify taxonomically challenging species. ${ }^{16}$ Previous literature showed that the ITS- 2 region has been used as a genetic marker for scrutinizing the diversity and exploring phylogenetic relationships among astigmatid mites. ${ }^{17}$ ITS- 2 marker has been used to successfully verify the taxonomy of closely related species and to differentiate and identify different species of astigmatid mites ${ }^{18,19}$ In this study, we used ITS-2 region to characterize astigmatid mites i.e. S. scabiei and house dust mites (HDMs) and then apply it as a phylogenetic marker to infer the genetic relationships among these two group of species.

\section{Materials and Methods Sample Collection}

The ethical approval for the collection of mites was granted by the Institutional Review Board (IRB) of Pir Mehr Ali Shah Arid Agriculture University Rawalpindi, Pakistan (letter No. PMAS-AAUR/ZOOL 362 approved on 22-3-2012). The S. scabiei mites were isolated from scabies infested patients visiting Military Hospital (MH), Rawalpindi after obtaining written informed consent from 20 individuals with ordinary scabies between September 2012 and October 2013. The cases of ordinary scabies were confirmed through clinical presentation, positive identification of mites and mite parts under the microscope.

For the collection of HDM, dust samples were collected from mattresses of beds and couches in the randomly selected houses and other places of human dwelling using vacuum cleaner. ${ }^{20}$ The saturated sodium chloride floatation method was used to isolate collected HDM from dust with supernatant filtered through $45 \mu \mathrm{m}$ filter paper. ${ }^{21}$ The astigmatid mites were then identified under the microscope. Both $S$. scabiei and HDM mites were then fixed in $70 \%$ ethanol and stored at $4^{\circ} \mathrm{C}$ until further processing.

\section{Extraction of Genomic DNA}

The genomic DNA of 20 individual astigmatid mites i.e. 10 of each Sarcoptes and HDM mites were extracted individually using the hotshot plus thermal shock technique. ${ }^{22}$ According to this method, $25 \mu$ l of alkaline lysis buffer ( $25 \mathrm{mM} \mathrm{NaOH}, 0.2 \mathrm{mM}$ disodium EDTA) was added in thermal-cycler tubes followed by three cycles of thermal shocks. Then $25 \mu \mathrm{l}$ neutralizing reagent $(40 \mathrm{mM}$ Tris- $\mathrm{HCl})$ was added to disrupt the exoskeleton and to adjust the $\mathrm{pH}$. The concentration of DNA was determined by nanodrop 2000 Spectrometer (Thermo Scientific, USA) at 260 and $280 \mathrm{~nm}$ wavelength.

\section{PCR Amplification and Sequencing}

The DNA of mites was amplified by using ITS-2 forward and reverse primers of sequence $5^{\prime}$ CGACTTTCGAACGCATATTGC $3^{\prime}$ and $5^{\prime}$ GCTTAAATTCAGGGGGTAATCTGC3' respectively. ${ }^{23,24}$ PCR was carried out in a final volume of $20 \mu \mathrm{l}$ with the following steps: pre-denaturation for $2 \mathrm{~min}$ at $95^{\circ} \mathrm{C}$, followed by 30 cycles of the $30 \mathrm{~s}$ at $94^{\circ} \mathrm{C}, 30 \mathrm{sec}$ at 56 ${ }^{\circ} \mathrm{C}, 50 \mathrm{sec}$ at $72{ }^{\circ} \mathrm{C} .8 \mu \mathrm{l} \mathrm{PCR}$ product of HDM was digested individually with restriction enzymes Hinf I $(10 \mathrm{U})$ and Taq I (20 U). Hinf I was incubated at $37^{\circ} \mathrm{C}$ and Taq I at $65^{\circ} \mathrm{C}$ overnight. The amplicons were visualized on $3 \%$ agarose gel, stained with ethidium bromide. The Big Dye ${ }^{\circledR}$ Terminator v3.1 cycle sequencing kit was used to directly sequence 10 purified PCR products in both directions by ABI 310 genetic analyzer (Applied Biosystems, Foster City, USA).

\section{Sequence Analysis}

ChromasPro (version 1.5) software (https://chromaspro.software.informer.com/1.5/) was used to edit and assemble the sequences. To verify results of sequenced samples, BLAST (https://blast.ncbi.nlm.nih.gov/Blast.cgi?PROGRAM =blastn\&PAGE_TYPE=BlastSearch\&LINK_LOC=blast home) was performed. The resulting top hit nucleotide sequences of ITS-2 were aligned using Clustalw (http://www.genome.jp/toolsbin/clustalw). The aligned sequences were further used to study and construct phylogenetic tree using Molecular Evolutionary Genetics Analysis (MEGA 7) based on Kimura-2-parameter (K2P) distance. ${ }^{25}$

\section{Results}

Genomic DNAs of S. scabiei and HDM mites were 
isolated from a total of twenty specimens and digested PCR products with both Hinf I and Taq I confirmed the morphologically identified $D$. farinae and no unexpected bands of PCR products or restriction fragments were observed.

The sequences of ITS-2 gene from S. scabiei and $D$. farinae showed $99 \%$ similarity respectively with whole genome of astigmatid mite (Accession no. AB778919.1 and GQ205600.1). The sequences obtained as a result of this study were submitted in GenBank under accession numbers KR010367, KJ409446, KT724354 and KT724355 for two S. scabiei samples (Sspak1, Sspak2) and two D. farinae samples (DerfPak1 and DerfPak2) respectively as shown in Table 1.

\begin{tabular}{|c|c|c|c|}
\hline $\begin{array}{c}\text { Mite } \\
\text { Species }\end{array}$ & Code & $\begin{array}{l}\text { Sequence } \\
\text { Length (bp) }\end{array}$ & $\begin{array}{l}\text { GenBank } \\
\text { Accession No }\end{array}$ \\
\hline \multirow[t]{2}{*}{ S. scabiei } & Sspak1 & 376 & KR010367 \\
\hline & Sspak2 & 359 & KJ409446 \\
\hline \multirow[t]{2}{*}{ D. farinae } & DerfPak1 & 338 & KT724354 \\
\hline & DerfPak2 & 335 & KT724355 \\
\hline
\end{tabular}

The length of the ITS- 2 sequences was between $335 \mathrm{bp}$ and $376 \mathrm{bp}$ for S. scabiei var. hominis and D. farinae respectively. ITS-2 sequences of astigmatid mites showed differences in terms of their length because of insertions and deletion at several sites. Three variable sites were determined for both astigmatid mites i.e. at 68,235 and 272 positions in ITS-2 sequence of S. scabiei and 97, 174 and 281 nucleotide positions in the ITS-2 sequences of $D$. farinae when compared with reported ITS-2 gene sequences in NCBI nucleotide database.

\begin{tabular}{|c|c|c|c|c|c|c|}
\hline & 1 & 2 & 3 & 4 & 5 & 6 \\
\hline $\begin{array}{l}\text { Der.f } \\
\text { (Japan) } \\
\text { Df-4 }\end{array}$ & $\begin{array}{l}0.000 \\
0.006\end{array}$ & & & & & \\
\hline DerfPak1 & 0.009 & 0.009 & & & & \\
\hline DerfPak2 & 0.012 & 0.013 & 0.003 & & & \\
\hline$D f-3$ & 0.025 & 0.025 & 0.016 & 0.019 & & \\
\hline$D f-1$ & 0.032 & 0.032 & 0.035 & 0.038 & 0.028 & \\
\hline$D f-2$ & 0.032 & 0.032 & 0.035 & 0.038 & 0.028 & 0.000 \\
\hline
\end{tabular}

Tandem repeats of nucleotides (AAA) ${ }_{n},(\text { TTT })_{n}$ and $(\text { GATT })_{n}$ were observed in the ITS-2 gene of both astigmatid mites are useful in determining

\begin{tabular}{|c|c|c|c|c|c|c|}
\hline & 1 & 2 & 3 & 4 & 5 & 6 \\
\hline SsPak1 & 0.000 & & & & & \\
\hline SsPak2 & 0.000 & & & & & \\
\hline Ss-1 & 0.003 & 0.003 & & & & \\
\hline Ss-2 & 0.005 & 0.005 & 0.008 & & & \\
\hline Ss-4 & 0.005 & 0.005 & 0.008 & 0.000 & & \\
\hline Ss-5 & 0.005 & 0.005 & 0.008 & 0.000 & 0.000 & \\
\hline Ss-3 & 0.005 & 0.005 & 0.008 & 0.000 & 0.000 & 0.000 \\
\hline
\end{tabular}

evolutionary percentage and for certain genealogical tests.

\section{Phylogenetic Analysis}

The phylogram constructed by using MEGA 7 software revealed two distinct clusters denoted by cluster I and cluster II (Fig 1) separating S. scabiei from the HDMS.

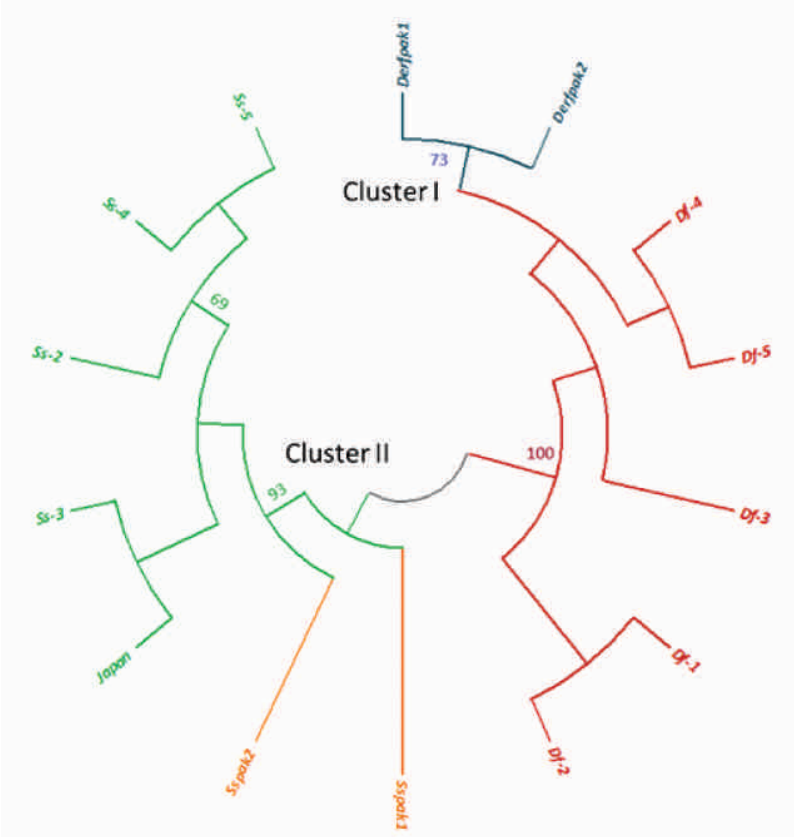

Fig 1: Phylogenetic relationship of S. scabiei and HDM mites based on ITS-2 sequences

The phylogenetic tree Cluster I includes $D$. farinae samples indicating close genetic similarity with bootstrap value of 100 . The Pakistani $D$. farinae (DerfPak1 and DerfPak2) lie within the HDM cluster, showing closer genetic relationship to each other than to the other HDMs included in the study (bootstrap value $=73$ ). Cluster II includes the Pakistani S. scabiei samples (SsPak1 and SsPak2) and other S. scabiei sequences with bootstrap value of 93. The phylogram showed an overall genetic 
distance of 0.1 indicating close genetic distance between HDM and Sarcoptes mites, indicating close genetic correlation.

\section{Pairwise Distance Calculation}

Pairwise distance was calculated on the basis of ITS-2 gene sequences of $D$ farinae and $S$. scabiei mites (Table 2 and Table 3). The values for genetic diversity were calculated within species for both S. scabiei and $D$. farinae separately. The values of genetic diversity for $D$. farinae range from 0.006-0.038 with an average of 0.022 . Similarly, the values of genetic diversity for S. scabiei range from 0.003-0.008 with an average of 0.004 . These values indicate that genes were genetically related with each other and there was little genetic diversity among them. The overall genetic distance between $S$. scabiei and D. farinae was calculated to be 0.53 .

\section{Discussion}

In the present study we characterized S. scabiei var. hominis sequences and compared them with other reported ITS-2 (mostly animals as a host) as previous studies on astigmatid mites did not include Sarcoptes mite from human host. ${ }^{23,26}$ Results of the studies carried out in Pakistan showed that ITS- 2 sequences of $D$. farinae is characterized by 3 transitions i.e. $Y, R$ and $W$ and of $S$. scabiei var. hominis sequences is characterized by two transitions and one transversion.

The comparison of ITS-2 sequences of astigmatid mites with sequences obtained from GenBank revealed that the sequences could not be used to differentiate geographical populations implying that the ITS-2 region was highly conserved in mites from different areas. ${ }^{26,27}$ The lower level of intraspecific variation and the relatively higher level of interspecific variation in ITS-2 region show that these genes may be suitable for studying the phylogeny of astigmatid mites. These species of mites were positioned in the phylogenetic trees according to their morphological characteristics. ${ }^{28}$

As reported by Yang et $\mathrm{al}^{27}$ the genetic distances between the species were higher as compared to distances within the species. ${ }^{26}$ The study revealed that ITS-2 region is suitable for phylogenetic studies of astigmatid mites as this region shows relatively lower variation within the species and higher variation between the different species of mites. These astigmatid mites were placed in the phylogenetic tree based on their genetic characteristics.

Our study with ITS-2 region is also supported by Suarez-Martinez et al. in which it has been established that ITS-2 region is not able to discriminate geographically isolated mite species. The mitochondrial $12 \mathrm{~S}$ rDNA is also not able to reflect differences between the families Pyroglyphidae and Acaridae ${ }^{29}$ because of sequence conservation the use of this marker is limited. In comparison, ITS-2 region is advantageous in studying population structure of astigmatid mites and can help us study low-level phylogenetic relationships between astigmatid mites. ${ }^{23,26}$

\section{Conclusion}

The present study is the first report from Pakistan using ITS-2 marker for the phylogenetic analysis of astigmatid mites. Although it is challenging to distinguishing the astigmatid mites based on their morphological characteristics, the ITS- 2 region can be for this purpose. However, ITS-2 region is not convenient for geographical discrimination of different species ${ }^{30}$ but it can be used to study lowlevel phylogenetic relationships between astigmatid mites.

\section{Acknowledgements}

The authors are thankful to Higher Education Commission, Islamabad, Pakistan for financial support under 5000 Indigenous Fellowship Program.

\section{REFERENCES}

1. Buczek A, Pabis B, Bartosik K, Stanislawek IM, Salata M, Pabis A. Epidemiological study of scabies in different environmental conditions in central Poland. Annals of epidemiology. 2006; 16: 423-8.

2. He Q, Ma Z, Dang X, Xu J, Zhou Z. Identification, diversity and evolution of MITEs in the genomes of microsporidian Nosema parasites. PloS one. 2015; 10: e0123170.

3. Lozano-Fernandez J, Tanner AR, Giacomelli M, Carton R, Vinther J, Edgecombe GD, et al. Increasing species sampling in chelicerate genomic-scale datasets provides support for monophyly of Acari and Arachnida. Nature communications. 2019; 10: 2295.

4. Colloff MJ, Stewart GA. House dust mites. Asthma. 1997; 2: 1089-103.

5. Pepato AR, da Rocha CE, Dunlop JA. Phylogenetic position of the acariform mites: sensitivity to homology assessment under total evidence. BMC Evolutionary Biology. 2010; 10: 235.

6. Wong SF, Chong AL, Mak JW, Tan J, Ling SJ, Ho TM. Molecular identification of house dust mites and storage mites. 
Experimental and applied acarology. 2011; 55: 123.

7. Dong X, Armstrong SD, Xia D, Makepeace BL, Darby AC, Kadowaki T. Draft genome of the honey bee ectoparasitic mite, Tropilaelaps mercedesae, is shaped by the parasitic life history. Gigascience. 2017; 6: gix008.

8. Carroll EL, Bruford MW, DeWoody JA, Leroy G, Strand A, Waits $L$, et al. Genetic and genomic monitoring with minimally invasive sampling methods. Evolutionary applications. 2018; 11: 1094-119.

9. Mounsey KE, Willis C, Burgess ST, Holt DC, McCarthy J, Fischer K. Quantitative PCR-based genome size estimation of the astigmatid mites Sarcoptes scabiei, Psoroptes ovis and Dermatophagoides pteronyssinus. Parasites \& vectors. 2012; 5 : 3.

10. Navajas M, Fenton B. The application of molecular markers in the study of diversity in acarology: a review. Experimental and applied acarology. 2000; 24: 751-74.

11. Skoracka A, Magalhaes S, Rector BG, Kuczyński L. Cryptic speciation in the Acari: a function of species lifestyles or our ability to separate species?. Experimental and Applied Acarology. 2015; 67: 165-82.

12. Beroiz B, Couso-Ferrer F, Ortego F, Chamorro MJ, Arteaga C, Lombardero $\mathrm{M}$, et al. Mite species identification in the production of allergenic extracts for clinical use and in environmental samples by ribosomal DNA amplification. Medical and veterinary entomology. 2014; 28: 287-96.

13. Arlian LG, Morgan MS. A review of Sarcoptes scabiei: past, present and future. Parasites \& vectors. 2017; 10: 297.

14. Cruickshank RH. Molecular markers for the phylogenetics of mites and ticks. Systematic and Applied Acarology. 2002; 7: 3-15.

15. Kane NC, King MG, Barker MS, Raduski A, Karrenberg S, Yatabe $\mathrm{Y}$, et al. Comparative genomic and population genetic analyses indicate highly porous genomes and high levels of gene flow between divergent Helianthus species. Evolution: International Journal of Organic Evolution. 2009; 63: 2061-75

16. Fukuyama S, Nishimura T, Yotsumoto H, Gushi A, Tsuji M, Kanekura $\mathrm{T}$, et al. Diagnostic usefulness of a nested polymerase chain reaction assay for detecting Sarcoptes scabiei DNA in skin scrapings from clinically suspected scabies. British Journal of Dermatology. 2010; 163: 892-4.

17. Feng S, Jiang M, Shi Y, Jiao K, Shen C, Lu J, et al. Application of the ribosomal DNA ITS2 region of Physalis (Solanaceae): DNA barcoding and phylogenetic study. Frontiers in plant science. 2016; 7: 1047.

18. Zhao LL, Feng SJ, Tian JY, Wei AZ, Yang TX. Internal transcribed spacer 2 (ITS 2) barcodes: A useful tool for identifying Chinese Zanthoxylum. Applications in plant sciences. 2018; 6: e01157.

19. Weeks AR, Opijnen TV, Breeuwer JAJ. AFLP fingerprinting for assessing intraspecific variation and genome mapping in mites. Experimental and Applied Acarology. 2000; 24: 77593.

20. Dautartiene A. Seasonal changes in house dust mites. Ekologija. 2001; 2:3-7.

21. Arlian LG, Woodford PJ, Bernstein IL, Gallagher JS. Seasonal population structure of house dust mites, Dermatophagoides spp. (Acari: Pyroglyphidae). Journal of Medical Entomology. 1983; 20: 99-102.

22. Alasaad S, Rossi L, Maione S, Sartore S, Soriguer RC, Pérez JM, et al. HotSHOT Plus ThermalSHOCK, a new and efficient technique for preparation of PCR-quality mite genomic DNA. Parasitology research. 2008; 103: 1455.

23. Noge K, Mori N, Tanaka C, Nishida R, Tsuda M, Kuwahara Y. Identification of astigmatid mites using the second internal transcribed spacer (ITS2) region and its application for phylogenetic study. Experimental \& applied acarology. 2005; 35: 29-46.

24. Wong SF, Chong AL, Mak JW, Tan J, Ling SJ, Ho TM. Molecular identification of house dust mites and storage mites. Experimental and applied acarology. 2011; 55: 123.

25. Kumar S, Stecher G, Tamura K. MEGA7: molecular evolutionary genetics analysis version 7.0 for bigger datasets. Molecular biology and evolution. 2016; 33: 18704.

26. Song Z, Wang X, Liang G. Species identification of some common necrophagous flies in Guangdong province, $S$ outhern China based on the rDNA internal transcribed spacer 2 (ITS2). Forensic Science International. 2008; 175: 17-22.

27. Yang B, Cai J, Cheng X. Identification of astigmatid mites using ITS2 and COI regions. Parasitology research. 2011; 108: 497-503.

28. Fain A. Adaptation, specificity and host-parasite coevolution in mites (Acari). International journal for parasitology. 1994; 24: 1273-83.

29. Suarez-Martinez EB, Montealegre F, Sierra-Montes JM, Herrera RJ. Molecular identification of pathogenic house dust mites using $12 \mathrm{~S}$ rRNA sequences. Electrophoresis. 2005; 26: 2927-34.

30. Alasaad S, Soglia D, Spalenza V, Maione S, Soriguer RC, Pérez JM, et al. Is ITS-2 rDNA suitable marker for genetic characterization of Sarcoptes mites from different wild animals in different geographic areas? Veterinary parasitology. 2009; 159: 181-5. 